\title{
Minoxidil suppressed mitochondrial depolarization and subsequent neuronal cell death by decreasing intracellular potassium level
}

\author{
Hiroshi Higashi, Miki Shintaku, Toshihiko Kinjo, Nobuyuki Kuramoto \\ Laboratory of Molecular Phamacology, Faculty of Pharmaceutical Sciences, Setsunan University, Japan
}

Intracellular potassium ion level is higher than extracellular space, which is regulated by basically sodium pump. Potassium ion generates a resting potential on the membrane by passing through the leak channels. Opening of the other potassium channels on the cell membrane induce hyperpolarizaion and it would cancel the excitation. Potassium channel are also on the mitochondrial inner membranes. Recent studies suggested that drugs, which open potassium channels in cellular and/or mitochondrial membrane protecr neuronal tissues against neurodegenerative situation including transient ischemia. Here, we have demonstrated whether Minoxidil, a potassium channel opener reduced damage on neuronal tissues that was caused by transient focal cerebral ischemia. Transient focal cerebral ischemia was induced in the 6 -weeks old male C57/BL6 mice by a 1-h middle cerebral artery occlusion (MCAO) of left hemisphere and subsequent reperfusion. Thereafter the mice were randomly divided into 4 groups, and either saline, edaravone $(6 \mathrm{mg} / \mathrm{kg} \mathrm{bw})$ or minoxidil ( 0.5 or $5 \mathrm{mg} / \mathrm{kg} \mathrm{bw}$ ) was intravenously injected, immediately. One day later, the brain was taken out, and 5 slices in total were prepared from each mouse with a thickness of $1 \mathrm{~mm}$ from the bregma, 2 on the front side and 3 on the back side, using a brain slicer, THe slices were stained with 2,3,5-Triphennyl tetrazolium chloride (TTC staining) to detect cellular respiration activity. Transient focal cerebral ischemia decreased TTC staining in ipsilateral striatal area by $50 \%$ compared to the opposite side, suggested that the operation damaged the neuronal tissues. The injection of edaravone immediately after the operation completely prevented the decrease. Asimilar prevention was observed with the injection with $5 \mathrm{mg} / \mathrm{kg}$ bw minoxidil instead of $0.5 \mathrm{mg} / \mathrm{kg} \mathrm{bw}$. The mechanism of action of minoxidil the open of ATPsensitive potassium channel, which is one of inward rectifying potassium channels. Minoxidil was once developed for the treatment of hypertension, but now it is famous as treatment for androgenic alopecia and it is applied for external application. We might need to think about injecting this medicine again in anticipation of the protective action of central nervous systems. 